When the Timing is Right: The Link Between Temporal Coupling in Dyadic Interactions and Emotion Recognition

Julia Bachmann ${ }^{1}$, Britta Krüger ${ }^{1}$, Johannes Keck ${ }^{1}$, Jörn Munzert ${ }^{1,2}$, and Adam Zabicki $^{1}$

${ }^{1}$ Neuromotor Behavior Laboratory, Department of Psychology and Sport Science, Justus Liebig University Giessen, Giessen, Germany

${ }^{2}$ Center for Mind, Brain and Behavior (CMBB), Philipps University of Marburg and Justus Liebig University Giessen, Germany

\begin{abstract}
Author Note
Julia Bachmann (1) https://orcid.org/0000-0001-9001-6505

Johannes Keck https://orcid.org/0000-0002-8035-0953

Adam Zabicki (1) https://orcid.org/0000-0002-0527-2705
\end{abstract}

Correspondence concerning this article should be addressed to:

Julia Bachmann

Kugelberg 62

35394 Giessen

GERMANY

julia.bachmann@sport.uni-giessen.de 


\begin{abstract}
Affective states can be understood as dynamic interpersonal processes developing over time and space. When we observe emotional interactions performed by other individuals, our visual system anticipates how the action will unfold. Thus, it has been proposed that the process of emotion perception is not only a simulative but also a predictive process - $\mathrm{a}$ phenomenon described as interpersonal predictive coding. The present study investigated whether the recognition of emotions from dyadic interactions depends on a fixed spatiotemporal coupling of the agents. We used an emotion recognition task to manipulate the actions of two interacting point-light figures by implementing different temporal offsets that delayed the onset of one of the agent's actions $(+0 \mathrm{~ms},+500 \mathrm{~ms},+1000 \mathrm{~ms}$ or $+2000 \mathrm{~ms})$. Participants had to determine both the subjective valence and the emotion category (happiness, anger, sadness, affection) of the interaction. Results showed that temporal decoupling had a critical effect on both emotion recognition and the subjective impression of valence intensity: Both measures decreased with increasing temporal offset. However, these effects were dependent on which emotion was displayed. Whereas affection and anger sequences were impacted by the temporal manipulation, happiness and sadness were not. To further investigate these effects, we conducted exploratory analyses of interpersonal movement parameters. Our findings complement and extend previous evidence by showing that the complex, noncoincidental coordination of actions within dyadic interactions results in a meaningful movement pattern and might serve as a fundamental factor in both detecting and understanding complex actions during human interaction.
\end{abstract}

Keywords: emotion recognition, dyadic interaction, temporal coupling, point-light displays, interpersonal predictive coding, simulation theory 


\section{When the Timing is Right: The Link Between Temporal Coupling in Dyadic Interactions and Emotion Recognition}

Social interactions are highly complex phenomena in which people rely on efficient mechanisms to derive information about each other. We are able to quickly retrieve a wealth of explicit and implicit information through the mere observation of a person. The human body, in particular, presents a rich and reliable source of nonverbal information. Body motion tells us not only what a person is doing but also why they are doing it. It communicates both the mental state of a person and their action demands to the perceiver (for a review, see de Gelder, 2006).

Individuals can quickly make a number of inferences about a person even if the available information is reduced to a minimum, as seen in point-light displays (PLD) in which shape information is removed while kinematic and configural information is preserved. It is not only possible to judge the gender or the identity of a person by simply observing these light points representing the kinematics of joints, we can also infer intentions and even affective states (Bachmann et al., 2020; Hill \& Pollick, 2000; Lorey et al., 2012; Mather \& Murdoch, 1994; Runeson \& Frykholm, 1983; Troje, 2002). This ability to retrieve relevant information allows us to generate adequate responses and integrate complex behavioral repertoires (de Gelder, 2006; de Gelder \& Hortensius, 2014). Interestingly, research shows that the recognition of affective states seems to be facilitated by observation of dyadic interactions rather than an individual's emotional display, highlighting the fact that affective states are not merely intrapersonal but dynamic interpersonal processes that develop over time and space (Clarke et al., 2005; Lorey et al., 2012).

It has been proposed that this "mind-reading" ability — that is, the ability to assign intentions or affective states from mere observation — can be explained by simulation theory (Gallese \& Goldman, 1998). Although simulation theory was thought to explain action understanding, it was soon extended and applied to intention and emotion understanding as 
well (Gallese, 2009; Rizzolatti \& Craighero, 2004). Within this framework, perceiving bodily expressions of emotion in others is thought to rely on one's own (motor) representations of these emotional movements and states (Gallese, 2009; Gallese et al., 2004). More specifically, we recognize another individual's emotional state by internally simulating the same emotional state in ourselves (Wicker et al., 2003).

However, it has also been proposed that action perception is not only a simulation of what is happening but also a prediction of what is going to happen (Kilner et al., 2007). This view is supported by behavioral evidence. In a seminal study, Neri et al. (2006) demonstrated that when interactive activity requiring close body contact is observed (e.g., fighting or dancing), the human visual system relies on the spatiotemporal coupling between the agents to retrieve information relating to each agent individually. More specifically, within meaningfully synchronized interactions, the actions of one agent serve as the predictor for the expected actions of the other agent. This is particularly true if actions of both agents are tightly coupled as in the context of fighting and dancing.

With respect to social interactions that do not require close body contact, Manera et al. (2011, 2013) and von der Lühe et al. (2016) demonstrated that observing dynamic communicative gestures of one agent enhances the visual discrimination of a second agent who is responding to this communicative gesture - a phenomenon that has been referred to as interpersonal predictive coding. Their results suggest that we possess implicit knowledge about the natural dynamics of human interaction that guides the processing of motion patterns generated by each of the two agents. Thus, when we observe actions performed by other individuals, we seem to anticipate how the action will unfold (von der Lühe et al., 2016). When we are engaged in a direct social interaction with a partner, prediction of the other person's actions helps us adjust our movements "online" (i.e., in real time) in order to plan an appropriate response (Becchio et al., 2010). However, up to now, little is known about how such a spatiotemporal relatedness of the actions of two agents is important for recognizing 
different emotions in social interactions. Here, we explicitly address two questions: (a) Does the recognition of emotions from dyadic interactions depend on a fixed spatiotemporal coupling of the actions of the individuals? (b) Is this coupling emotion-specific?

To address these questions, we employed an emotion recognition task in which we manipulated the actions of two interacting agents by implementing different temporal offsets. More specifically, we delayed the onset of one of the agent's actions by either $+0 \mathrm{~ms},+500$ ms, $+1000 \mathrm{~ms}$, or $+2000 \mathrm{~ms}$. Participants were asked to determine the subjective valencethat is, how positive or negative a stimulus is perceived to be-as well as the emotion category (happiness, anger, sadness, or affection) displayed within a given scene. In light of experimental evidence demonstrating that spatiotemporally congruent actions are critical for the perception of social interactions, we anticipate that recognition will be impaired as a result of the temporal offset (Manera et al., 2011; Neri et al., 2006; Petrini et al., 2014; Thurman \& Lu, 2014). Furthermore, we expect that because some emotions have a stronger interpersonal character (e.g., affection is characterized by stronger bodily interaction than sadness), the recognition of certain emotions will be affected more strongly by the temporal decoupling (Clarke et al., 2005). Finally, we aimed to explore whether objective movement parameters are influenced by the temporal offset, and how these potential changes correspond to the subjective measure of emotion perception. Therefore, we quantified objective interpersonal movement features of the observed scenes and related these to recognition performance.

\section{Methods}

\section{Participants}

Forty-four adults (19 females and 25 males, mean age $=24.68$ years, $S D=6$ years) with normal or corrected-to-normal vision participated in the study. None reported any history of psychiatric or neurological disorders, and they had no history or current use of psychoactive medication. All subjects gave written informed consent in accordance with the Declaration of Helsinki. The protocol was approved by the local ethics committee. We used 
the German version of the Emotional Competence Questionnaire (Emotionale-KompetenzFragebogen, EKF; Rindermann, 2009) to assess the participant's score in four abilities. To control for the participant's emotional state, we applied the State-Trait Anxiety Inventory (STAI; Spielberger et al., 1983) and the Beck Depression Inventory (BDI-II; Beck et al., 1996). STAI-X1 scores ranged from 21 to $56(M=33.91, S D=7.43)$; STAI-X2, from 20 to $70(M=38.39, S D=11.10)$, with greater scores indicating greater anxiety. BDI-II scores ranged from 0 to $48(M=8.40, S D=8.30)$. Although some of these participants showed clinically relevant scores (i.e., a BDI-II total score above 13), this did not affect their emotion recognition performance (see Supplementary Material, Figure S1).

\section{Stimuli}

Point-light displays of emotional interactions were taken from a motion-capture data set created by Bachmann et al. (2020). In brief (for full details see Bachmann et al., 2020), eight pairs of nonprofessional actors were asked to portray the following four emotional states within an interaction: happiness, sadness, anger, and affection. Each emotional state was acted out in varying intensities in order to obtain a variety of emotional expressions. Actors were asked to perform intuitively within the context of the given situation, thereby allowing freedom and subsequently variability in their expression. Moreover, both actors were instructed specifically to act out the same emotion in order to produce a congruent behavioral pattern. Interactions were recorded with an optical motion capture system (Vicon Motion Systems, Oxford, England). Next, video files of 4-s sequences were created from the original coordinate 3D (C3D) files using MATLAB software (MathWorks, Natick, MA). The final stimuli consisted of two point-light figures, each consisting of 15 markers indicating the major joints of each actor (head, clavicle, shoulders, elbows, wrists, hips, knee, and ankles). In the next step, recognizability was tested within a pilot study. Eighteen independent participants who were naive to the point-light sequences were asked to evaluate the valence 
and the emotional category of the interactions. We then excluded ambiguous scenes from the dataset.

From this dataset, we randomly selected 68 point-light interactions (i.e., 17 scenes per emotion category: affection, anger, happiness, sadness) to create the final stimulus set. Using MATLAB software (MathWorks, Natick, MA), four different temporal conditions were assembled per sequence. In the no offset condition (+0 ms), the actions of the actors were displayed in their original form. All other conditions contained temporal offsets between the actions of the agents. Within these conditions, the onset of the actions of one randomly selected agent was delayed by either $500 \mathrm{~ms}(+500 \mathrm{~ms}), 1000 \mathrm{~ms}(+1000 \mathrm{~ms})$, or $2000 \mathrm{~ms}$ (+2000 ms). Finally, all sequences were cut down from 4 to $2 \mathrm{~s}$ in order to obtain overlapping actions (see Fig 1A).

\section{Apparatus and Procedure}

Stimuli were displayed on a 17.3-inch full HD screen (display resolution: 1,920 x 1,080; refresh rate $60 \mathrm{~Hz}$ ) using MATLAB (MathWorks, Natick, MA) software. Participants were seated at a viewing distance of approximately $60 \mathrm{~cm}$ from the screen. The dots of the point-light sequences were light grey against a black background.

Prior to the experiment, participants were familiarized with the task and subsequently performed a test run. In the test run, they categorized four different emotional interactions. They were presented with eight point-light interactions and subsequently had to judge (a) the valence of the scene and (b) the emotional category that it displayed (i.e., sadness, anger, happiness, or affection). All scenes were depicted in their original temporal order - that is, with no temporal manipulation.

The actual experiment comprised a series of 272 sequences ( 4 emotion categories $\times 17$ point-light sequences $\times 4$ temporal offsets). Each sequence was presented once, except for the no-offset condition $(+0 \mathrm{~ms})$ that was presented twice in order to obtain an individual baseline 
for each participant. This resulted in a total of 340 trials. Sequences were displayed in a pseudorandomized order to prevent the same trial from being presented twice consecutively.

A forced-choice paradigm was employed: Each trial started with a fixation phase $(1 \mathrm{~s})$, followed by a stimulus sequence ( $2 \mathrm{~s}$ ) and two ratings (see Fig 1B). In the first rating, participants were asked to judge the subjectively perceived valence of the interaction on an 11-point scale ranging from -5 (extremely negative) to 5 (extremely positive) with 0 (neutral) marking the center of the scale. Next, participants were asked to assign an emotional category to the observed sequences (i.e., affection, anger, happiness, or sadness). They were instructed to make intuitive judgments; however, they were not restricted in their rating time. In total, the experimental session took about 45 min per participant.

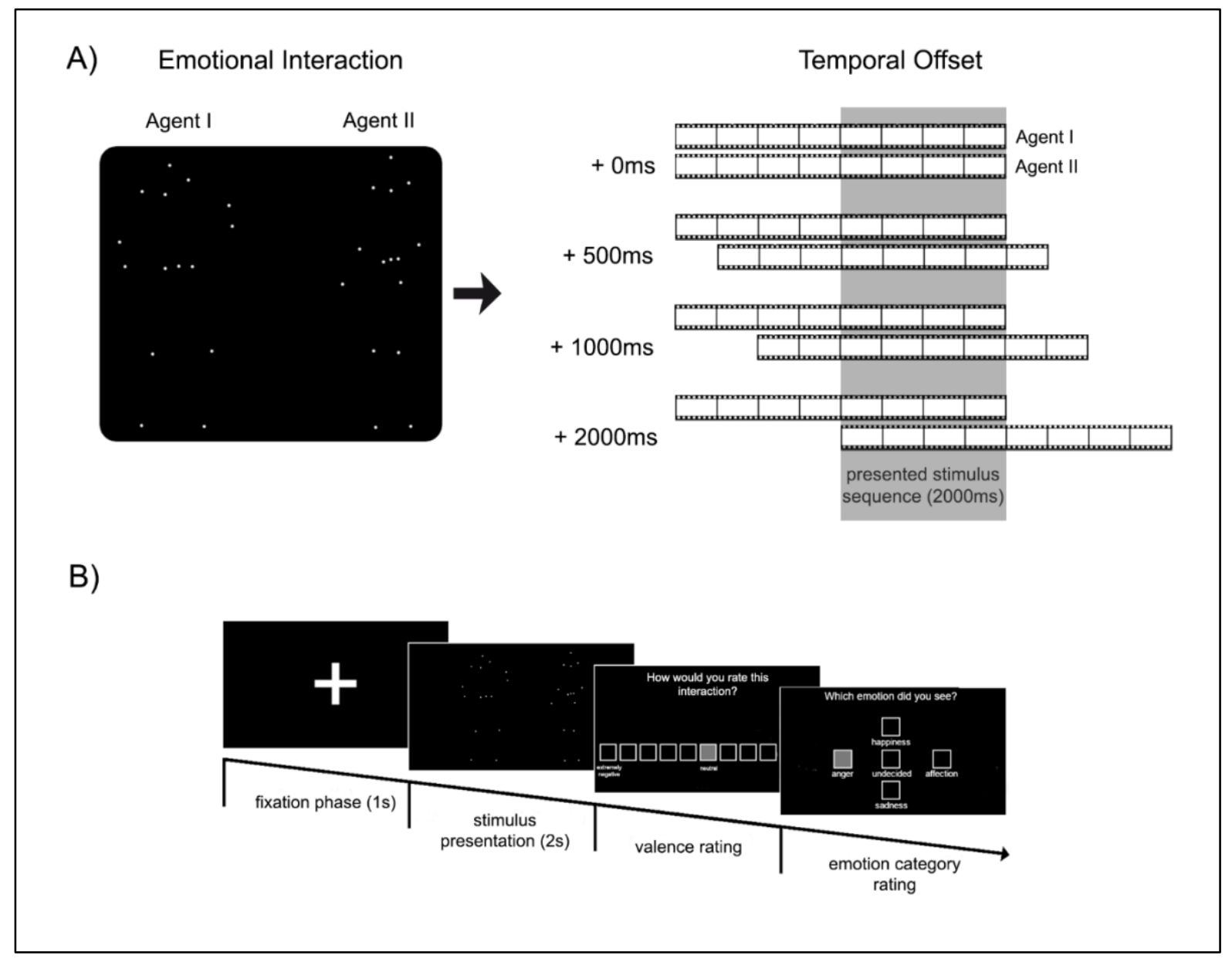

Figure 1: Stimulus creation and experimental timeline. A) Point-light displays of emotional interactions were taken from a motion-capture data set created by Bachmann et al. (2020). Next, temporal offsets $(+0 \mathrm{~ms},+500 \mathrm{~ms},+1000 \mathrm{~ms},+2000 \mathrm{~ms})$ were implemented, and the 
duration of stimulus sequences was reduced to $2000 \mathrm{~ms}$ in total. B) Temporal structure of one trial. Each trial started with a fixation phase (1s) followed by the stimulus sequence $(2 \mathrm{~s})$. Subsequent to the stimulus presentation, a valence as well as emotion category rating was employed with no maximum response time.

\section{Data Analysis and Statistics}

\section{Recognizability of emotional interactions}

In the first step of our analyses, we calculated a confusion matrix by comparing the emotion category of baseline sequences (i.e., first presentation of each +0 ms interaction) with the category ratings from the validation study. To ensure a sufficient degree of recognizability for the stimuli, we tested the recognition rates of each emotional category against chance (25\%) using a Wilcoxon signed-ranks test. Following Rosenthal (1994), we calculated effect sizes $r$ as the $Z$ statistic divided by the square root of the sample size $N$, with $N$ being the total number of participants.

\section{Influence of temporal decoupling on emotion recognition accuracy}

In the next step, we explored the effect of different temporal offsets on recognition performance. We used the emotion category rating of the first presentation of each $+0 \mathrm{~ms}$ sequence as an individual baseline measure (in contrast to the previous analysis, in which validation data were used as a baseline). On the basis of this individual baseline, we calculated relative recognition accuracies by comparing the category ratings of the remaining sequences containing different temporal offsets (i.e., $+0 \mathrm{~ms},+500 \mathrm{~ms},+1000 \mathrm{~ms},+2000 \mathrm{~ms}$ ), to the respective baseline rating.

Due to a violation of the normal distribution assumption, we applied a two-step relative rank transformation according to Templeton (2011) to normalize our data. Finally, we calculated a 4 (emotion category: affection, anger, happiness, sadness) $\times 4$ (temporal offsets: $+0 \mathrm{~ms},+500 \mathrm{~ms},+1000 \mathrm{~ms},+2000 \mathrm{~ms}$ ) repeated measures ANOVA for the dependent variable recognition performance. 
We calculated sensitivity $\left(d^{\prime}\right)$ values for each emotion category and temporal offset. More specifically, we calculated the proportion of hits (when the displayed emotion category was consistent with that identified) and false alarms (when the identified emotion category was not consistent with the one displayed) for each participant. Proportions of 0 were replaced with $0.5 / N_{\mathrm{p} / \mathrm{a}}$, and proportions of 1 were replaced with $\left(N_{\mathrm{p} / \mathrm{a}}-0.5\right) / N_{\mathrm{p} / \mathrm{a}}$ (in which $N_{\mathrm{p}}$ is the number of baseline sequences that were identified as a specific emotion category by a participant, and $N_{\mathrm{a}}$ the number of the remaining baseline sequences not identified as the respective category: i.e., $N_{\mathrm{a}}=68-N_{\mathrm{p}}$ ). Finally, we calculated a 4 (emotion category:

affection, anger, happiness, sadness) $\times 4$ (temporal offsets: $+0 \mathrm{~ms},+500 \mathrm{~ms},+1000 \mathrm{~ms},+2000$ ms) repeated measures ANOVA using $d$ 'values.

\section{Influence of temporal decoupling on valence judgment}

Next, we explored the effect of different temporal offsets on subjective valence perception by calculating a 4 (emotion category: affection, anger, happiness, sadness) $\times 4$ (temporal offsets: $+0 \mathrm{~ms},+500 \mathrm{~ms},+1000 \mathrm{~ms},+2000 \mathrm{~ms}$ ) repeated measures ANOVA for the dependent variable valence intensity.

\section{Movement features and emotion recognition}

In a last step, we performed exploratory analyses to elucidate how specific quantitative movement features related to the recognizability of the emotional sequences. First, we used the SAMI toolbox (Similarity Analysis of Human Movements and Interactions, see Zabicki \& Keck, 2021) to calculate relevant movement features of the interaction sequences. This enabled us to identify changes in interpersonal movement parameters within the stimuli caused by the introduced temporal offset. Specifically, we determined 12 parameters describing the interaction of the observed two agents (see Supplementary Table S1). These features included, for instance, interpersonal distance (i.e., the average distance between the two agents), personal space (i.e., the time each agent spent in the personal space of the interactive partner), and socalled motion energy balance. Motion energy balance is a parameter providing information 
about how much of the total amount of body movement over time can be ascribed to each interactive partner. A perfectly balanced motion energy (equals a maximum value of 1) would be characterized by two agents who each take up the same amount of the total motion energy presented in the sequence.

Each feature was calculated for each interaction sequence (68 in total) and for each temporal offset $(+0 \mathrm{~ms},+500 \mathrm{~ms},+1000 \mathrm{~ms},+2000 \mathrm{~ms})$. Next, we conducted a one-way repeated measures ANOVA with temporal offset as the within factor to find out whether the kinematic parameters were altered significantly by the temporal offset. We did this only for those emotion categories that showed diminished recognition accuracies caused by the temporal offset, because our aim was to elucidate whether decreased recognition accuracies could potentially be explained by changes of movement features due to the implemented temporal offset.

In case a kinematic parameter would prove to be modulated in an analogous way as the corresponding subjective recognition accuracy, we conducted further analyses to see how the change in kinematic features related to emotion recognition. We operationalized this by sorting movement parameters on the basis of whether the sequence was correctly assigned to its emotional category (based on the individual baseline rating). This was done for each trial per participant, again only for the emotion categories of interest. In a next step, we averaged movement features of both categories (correctly vs. incorrectly identified) across trials for each participant. Finally, we conducted a nonparametric Wilcoxon signed-ranks test to test for a significant difference between the categories. Effect size was calculated by means of a matchedpairs rank biserial correlation.

\section{General procedure}

In case of violations of the normal distribution, we applied a two-step relative rank transformation according to Templeton (2011) to normalize our data when applying parametric tests. All recognition rates are presented in percentages. The respective statistics 
rely on normalized data. We report Huyn-Feldt- or Greenhouse-Geisser-corrected $p$ values in order to counteract observed violations of sphericity. Moreover, all post hoc pairwise comparisons were Bonferroni-corrected.

\section{Results}

\section{Recognizability of Emotional Interactions}

Overall, the emotion category ratings of the baseline interactions were consistent with the categories identified within the validation study, as displayed by the confusion matrix in Figure 2A. The Wilcoxon signed-ranks test revealed that sequences of each emotional category were classified significantly above chance level (affection: $Z=5.78, p<.001, r=$ .87 ; anger: $Z=5.81, p<.001, r=.88$; happiness $Z=5.79, p<.001, r=.87$; sadness: $Z=5.79$, $p<.001, r=.87$; see Figure 2B). Anger sequences were categorized with the highest accuracy, displaying a mean recognition performance of $88 \%$. The second most accurately categorized emotion was happiness at $76 \%$. This was followed by sadness with a mean recognition performance of $68 \%$. Affection, however, showed a markedly lower recognition performance of $58 \%$, yet still within a fully satisfactory range.

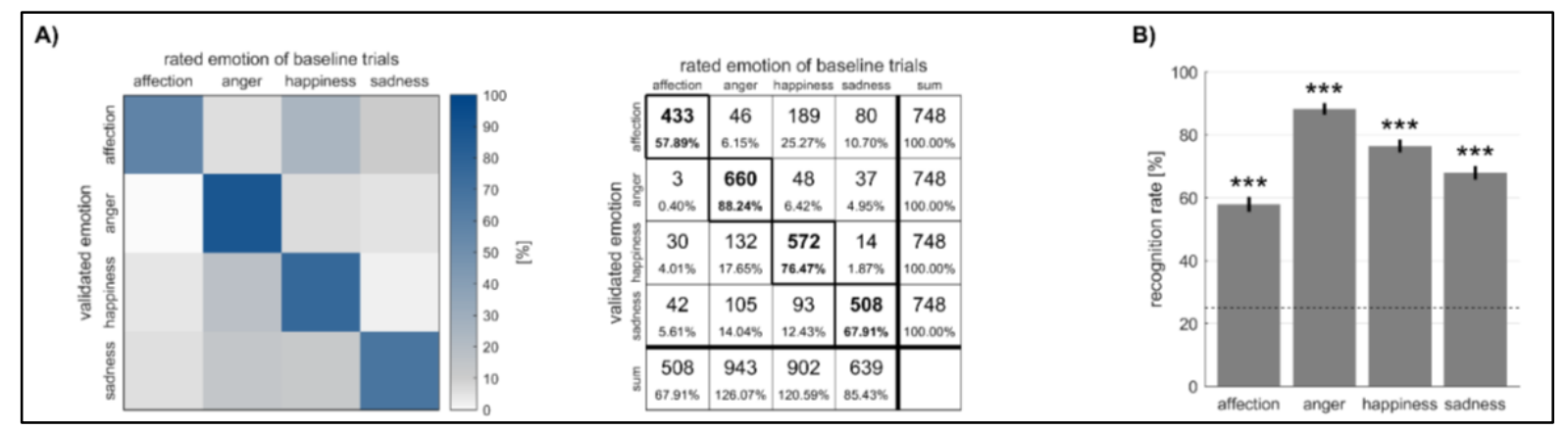

Figure 2: Confusion matrix and recognition rates. A) Confusion matrix displaying number and percentage of participants' baseline emotion category ratings compared with the validated emotion category rating. B) Emotion recognition rates of baseline interactions for all emotion categories (i.e., affection, anger, happiness, sadness). Bars (and their standard errors) show average emotion recognition rate in \%. Significance level is indicated by asterisks $(* * * \mathrm{p}<$ $.001)$. Dashed line represents threshold of chance recognition (25\%). 


\section{Temporal Decoupling and Emotion Recognition}

In the following, the respective statistics rely on normalized data, and all descriptive data are presented in actual values. We first investigated whether the recognition of emotional interactions was affected by temporal offsets. Results revealed a main effect of temporal offset, $F(3,129)=30.531, p<.001, \eta_{\mathrm{p}}^{2}=0.42$. Bonferroni-corrected post hoc analyses showed that recognition rates for the $+0 \mathrm{~ms}$ condition were significantly higher than for all other conditions (all $p<.01$ ). Recognition rates decreased linearly from the $+0 \mathrm{~ms}$ to the +2000 ms interaction sequences. However, there was no significant difference between +1000 $\mathrm{ms}$ and $+2000 \mathrm{~ms}$ (see Figure 3A). Moreover, we found a main effect of emotion category, $F(2.75,118.12)=7.75, p<.001, \eta_{\mathrm{p}}^{2}=0.15$. Post hoc analyses indicated that affection scenes had significantly lower recognition rates than any of the other emotions (all $p<.001$ ). Recognition rates for anger, happiness, and sadness scenes did not differ from each other (all $p>.05)$.

There was a significant interaction between temporal offset and emotion category, $F(9,387)=3.79, p<.001, \eta_{\mathrm{p}}^{2}=0.04$. Considering each emotion category separately, results showed that for affection scenes, the recognition rates between $+0 \mathrm{~ms}$ and $+500 \mathrm{~ms}$ did not differ significantly $(p=0.08)$. However, recognition rates for $+0 \mathrm{~ms}$ sequences were significantly higher compared to $+1000 \mathrm{~ms}$ and $+2000 \mathrm{~ms}$ interactions (all $p<.001$ ). With respect to anger scenes, recognition rates decreased linearly from the $+0 \mathrm{~ms}$ to the $+2000 \mathrm{~ms}$ interaction sequences. Post hoc comparisons showed that all temporal offsets differed significantly from each other (all $p<.05)$. Post hoc analyses showed no differences between the temporal offset conditions for happiness and sadness interactions (all $p>.05$ ) (see Figure 3B). 


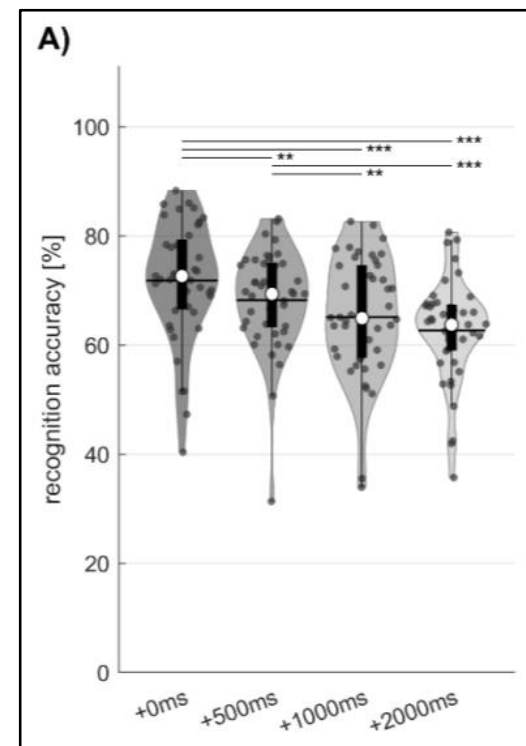

B)

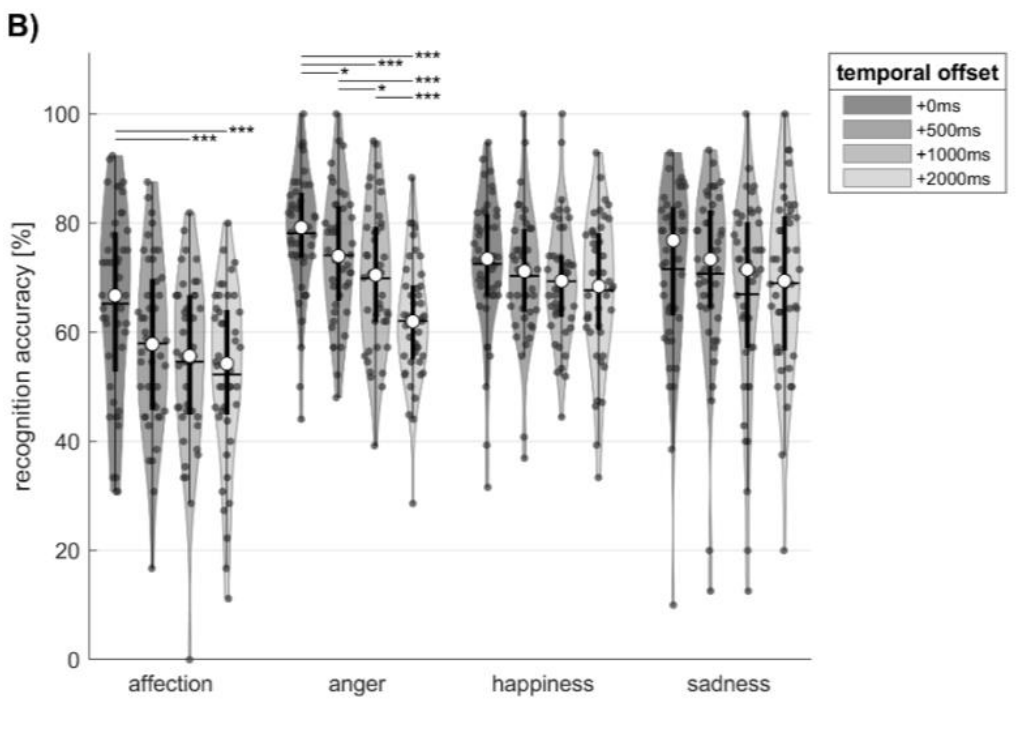

Figure 3: Influence of temporal offset on recognition performance. Emotion recognition accuracies (in \%) for A) all four temporal offsets (+0 ms, +500 ms, +1000 ms, +2000 ms) and then B) additionally separated by emotion category (affection, anger, happiness, sadness). Violin plots display the distribution of individual performances (single colored dots). Each violin plot contains information about the median (i.e., white dot), the 25th and 75th percentiles (i.e., bold vertical line), and the mean (i.e., horizontal line). Horizontal lines between variables indicate significance of difference between temporal offsets. Significance level is indicated by asterisks $(* p<.05 ; * * p<.01 ; * * *<.001)$.

To explore the interaction between temporal offset and emotion category, we calculated sensitivity $\left(d^{\prime}\right)$ values for each emotion category and temporal offset. We found a main effect of temporal offset, $F(3,129)=37.21 ; p<.001, \eta_{\mathrm{p}}^{2}=0.46$, with $d^{\prime}$ 'decreasing linearly from the $+0 \mathrm{~ms}$ to the $+2000 \mathrm{~ms}$ temporal offset condition (all $p<.001$ ) (see Figure 4A). Moreover, we found a main effect of emotion category, $F(2.75,118.12)=7.75, p<$ $.001, \eta_{\mathrm{p}}^{2}=0.15$, indicating significantly lower values for happiness sequences compared to anger $\left(M_{\text {diff }}=0.21, p<.01,95 \% \mathrm{CI}[.04, .39]\right)$ and sadness $\left(M_{\text {diff }}=0.28, p<.001,95 \% \mathrm{CI}\right.$ $[.11, .46])$ sequences. Finally, we found a significant interaction between temporal offset and emotion category, $F(8.19,352.01)=2.12, p<.05, \eta_{\mathrm{p}}^{2}=0.05$. Post hoc tests showed that for affection sequences, $d$ ' values did not differ significantly between $+0 \mathrm{~ms}$ and $+500 \mathrm{~ms}$. However, the $+0 \mathrm{~ms}$ differed significantly from the $+1000 \mathrm{~ms}$ and $+2000 \mathrm{~ms}$ sequences (all $p$ $<.05)$. For anger scenes, we found that $d$ ' values were significantly higher for the $+0 \mathrm{~ms}$ sequences than for all other sequences (all $p<.001$ ). Moreover, $\mathrm{d}^{\prime}$ values for the $+500 \mathrm{~ms}$ 
sequences differed significantly from $d$ ' values for the $+2000 \mathrm{~ms}$ sequences $\left(M_{\text {diff }}=0.26, p<\right.$ $.001,95 \%$ CI $[.09, .43])$. For happiness sequences, post hoc analysis indicated that $d$ ' values were significantly higher for the +0 ms sequences than for all other sequences (all $p<.05$ ). Moreover, $d$ ' values for the +500 ms sequences were significantly greater than for the +1000 ms and $+2000 \mathrm{~ms}$ sequences (all $p<.05$ ). Finally, post hoc tests indicated that for sadness trials, $d$ ' values were significantly greater for $+0 \mathrm{~ms}$ sequences than for $+1000 \mathrm{~ms}$ and +2000 ms sequences (all $p<.05)$ but not for $+500 \mathrm{~ms}$ sequences $(p=.62)$ (see Figure 4B).

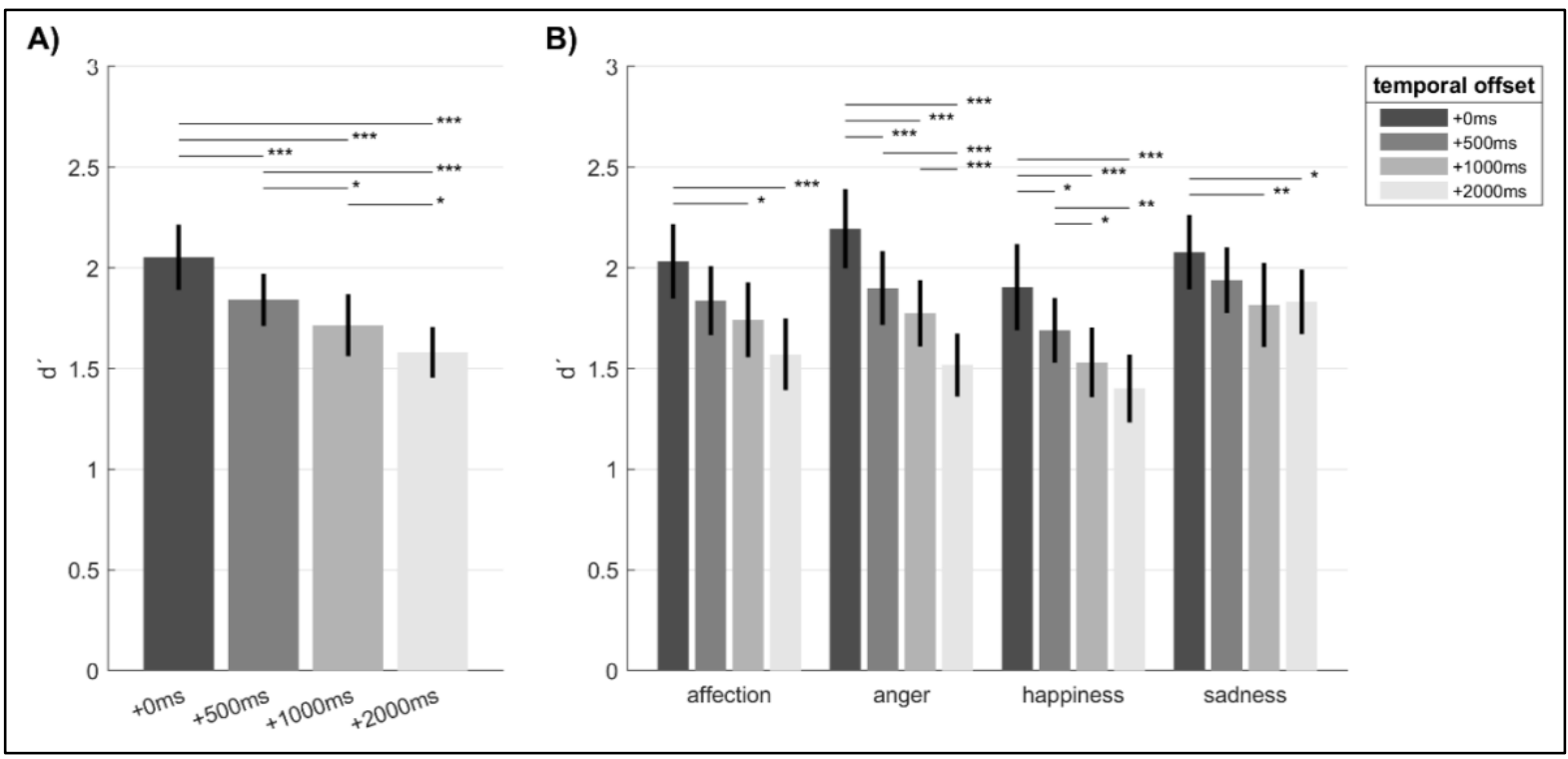

Figure 4: Influence of temporal offset on recognition sensitivity. d' values for A) all four temporal offsets ( $+0 \mathrm{~ms},+500 \mathrm{~ms},+1000 \mathrm{~ms},+2000 \mathrm{~ms}$ ) and B) additionally separated by emotion category (affection, anger, happiness, sadness). Bars (and their standard errors) show average d' values. Horizontal lines indicate significant differences between temporal offsets. Significance level is indicated by asterisks $(* p<.05 ; * * p<.01 ; * * *<.001)$.

\section{Temporal Decoupling and the Perception of Valence Intensity}

We further investigated whether the perception of valence intensity was affected by temporal offsets. Again, our results revealed a main effect of temporal offset, $F(2.47,106.41)$ $=6.78, p<.001, \eta_{\mathrm{p}}^{2}=0.14$. Bonferroni-corrected post hoc analyses showed that valence ratings did not differ between the $+0 \mathrm{~ms}$ and $+500 \mathrm{~ms}$ condition $\left(M_{\text {diff }}=0.03, p=1\right)$. However, valence ratings in the $+0 \mathrm{~ms}$ condition were judged to be more intense (i.e., absolute valence $)$ compared to the $+1000 \mathrm{~ms}\left(M_{\text {diff }}=0.09, p<.01,95 \%\right.$ CI $\left.[.02, .16]\right)$ and 
$+2000 \mathrm{~ms}\left(M_{\text {diff }}=0.08, p<.05,95 \%\right.$ CI $\left.[.01, .15]\right)$ conditions. Moreover, valence ratings within +500 ms sequences were judged to be significantly more intense than $+1000 \mathrm{~ms}$ sequences $\left(M_{\text {diff }}=.06, p<.01,95 \%\right.$ CI $\left.[.02, .10]\right)($ see Figure $5 \mathrm{~A})$.

Additionally, we found a main effect of emotion category, $F(3,129)=36.81, p<.001$, $\eta_{\mathrm{p}}^{2}=0.46$. Post hoc analyses indicated that happiness received the significantly highest valence ratings of all emotion categories (all $p<.001$ ). In contrast, sadness received the lowest valence ratings of all categories (all $p<.001$; see Figure 5B). Finally, we found an interaction between temporal offset and emotion category, $F(9,387)=2.02, p<.05, \eta_{\mathrm{p}}^{2}=.05$. Post hoc analyses showed that for affection, valence intensity ratings were significantly higher for +0 ms sequences compared to $+2000 \mathrm{~ms}$ sequences $\left(M_{\text {diff }}=.19, p<.05,95 \%\right.$ CI $\left.[.01, .36]\right)$. With respect to anger sequences, we found significantly greater intensity ratings for $+0 \mathrm{~ms}$ sequences compared to $+1000 \mathrm{~ms}$ sequences $\left(M_{\text {diff }}=.16, p<.01,95 \%\right.$ CI $\left.[.05, .27]\right)($ see Figure 5C). 


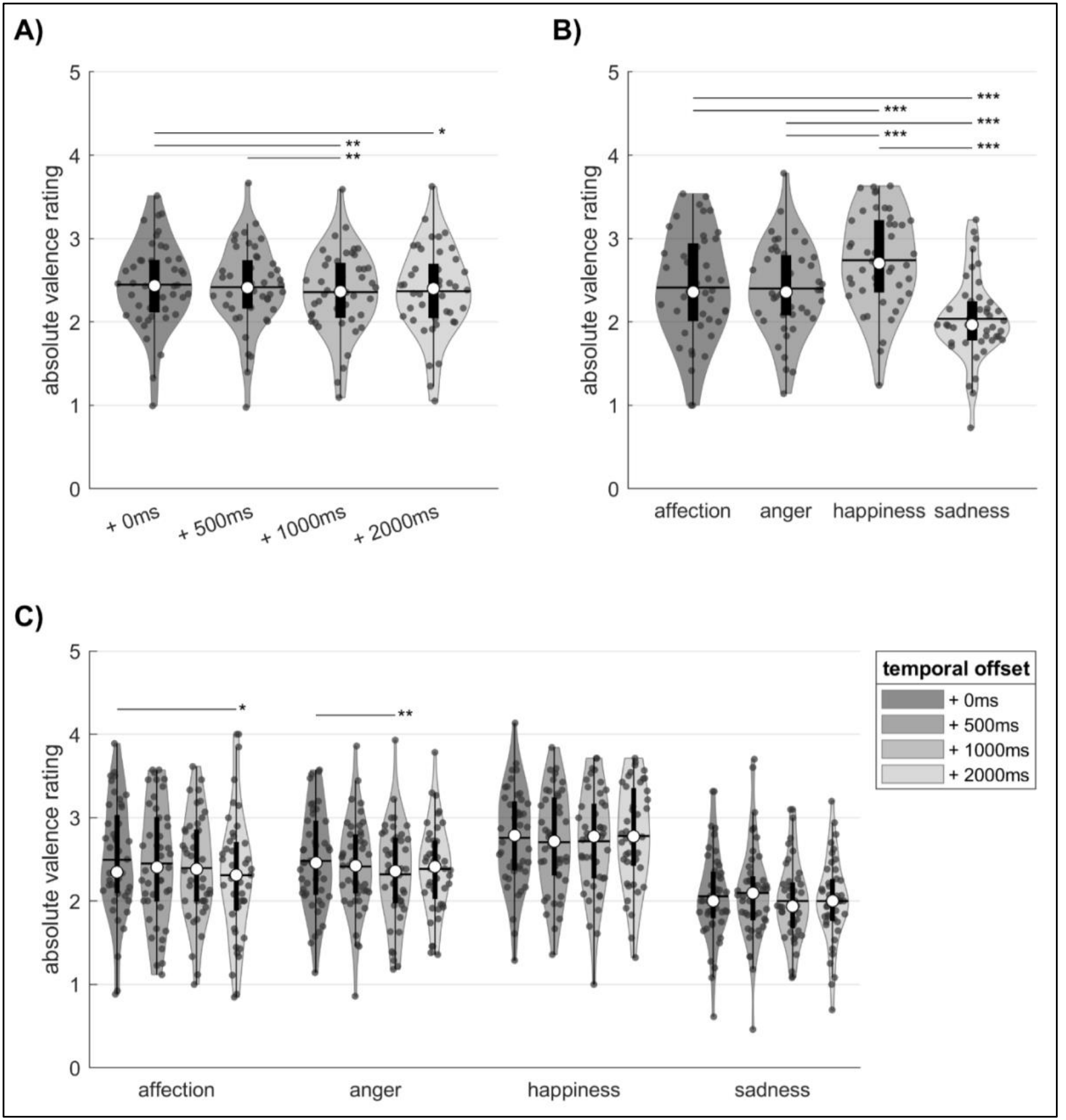

Figure 5: Influence of temporal offset on subjective valence perception. Absolute valence rating for A) all four temporal offsets $(+0 \mathrm{~ms},+500 \mathrm{~ms},+1000 \mathrm{~ms},+2000 \mathrm{~ms})$; and B) all four emotion categories (affection, anger, happiness, sadness); and C) per emotion category separated by temporal offset. Violin plots display the distribution of individual performances (single colored dots). Each violin plot contains information about the median (i.e., white dot), the 25th and 75th percentiles (i.e., bold vertical line), and the mean (i.e., horizontal line). Horizontal lines between variables indicate significance of difference between temporal offsets. Significance level is indicated by asterisks $\left({ }^{*} p<.05 ; * * p<.01 ; * * * p .001\right)$.

\section{Associations Between Movement Features and Emotion Recognition}

Results showed that the temporal decoupling led to a decrease in recognition performance exclusively for affection and anger sequences. Therefore, we explored whether 
movement patterns of affection and anger sequences were also affected systematically by the temporal offset. With respect to affection sequences, repeated measures ANOVA revealed a significant effect for the parameter motion energy balance. For anger sequences, in contrast, we found effects for the parameters average interpersonal distance and personal space.

\section{Motion energy balance}

In affection sequences, we found a significant main effect for motion energy balance, $F(1.64,26.17)=4.832, p<.05, \eta_{\mathrm{p}}^{2}=.23$, indicating that this was significantly impacted by the temporal offset. Bonferroni-corrected post hoc tests indicated a decline in motion energy balance for an increased temporal offset. However, differences failed to attain the significance level of $\mathrm{p}<.05$ (post hoc comparison of $+0 \mathrm{~ms}$ sequences with $+2000 \mathrm{~ms}$ sequences resulted in: $M_{\text {diff }}=.11, p=.074,95 \%$ CI [-.008, .232], see Figure 6A).

A Wilcoxon signed-ranks test revealed that the motion energy balance was significantly greater for correctly $(M=.84, S E M=.004)$ compared to incorrectly identified $(M=.81, S E M=.006)$ sequences, $\mathrm{Z}=3.26, \mathrm{p}<.01, \mathrm{r}=.56$ (see Figure $6 \mathrm{D})$, indicating that higher motion energy balance seemed to enhance recognition of affection sequences.

\section{Average interpersonal distance}

For anger sequences, we found effects for average interpersonal distance and personal space. With respect to average interpersonal distance, there was a main effect of temporal offset, $F(3,48)=4.168, p<.05, \eta_{\mathrm{p}}^{2}=.21$. Bonferroni-corrected post hoc tests indicated significantly greater distances for a temporal offset of $+1000 \mathrm{~ms}$ compared to a temporal offset of $+2000 \mathrm{~ms}\left(M_{\text {diff }}=105.64, p<.05,95 \%\right.$ CI $[7.36,203.93]$ (see Figure 6B).

\section{Personal space}

We also found a main effect of temporal offset for personal space in anger sequences, $F(1.95,31.12)=3.81, p<.05, \eta_{\mathrm{p}}^{2}=.19$. Bonferroni-corrected post hoc tests indicated significantly less time spent in the personal space of the other agent for a temporal offset of 
$+1000 \mathrm{~ms}$ compared to a temporal offset of $+2000 \mathrm{~ms}\left(M_{\text {diff }}=-13.03, p<.05,95 \%\right.$ CI [-

25.63, -0.43], see Figure 6C).

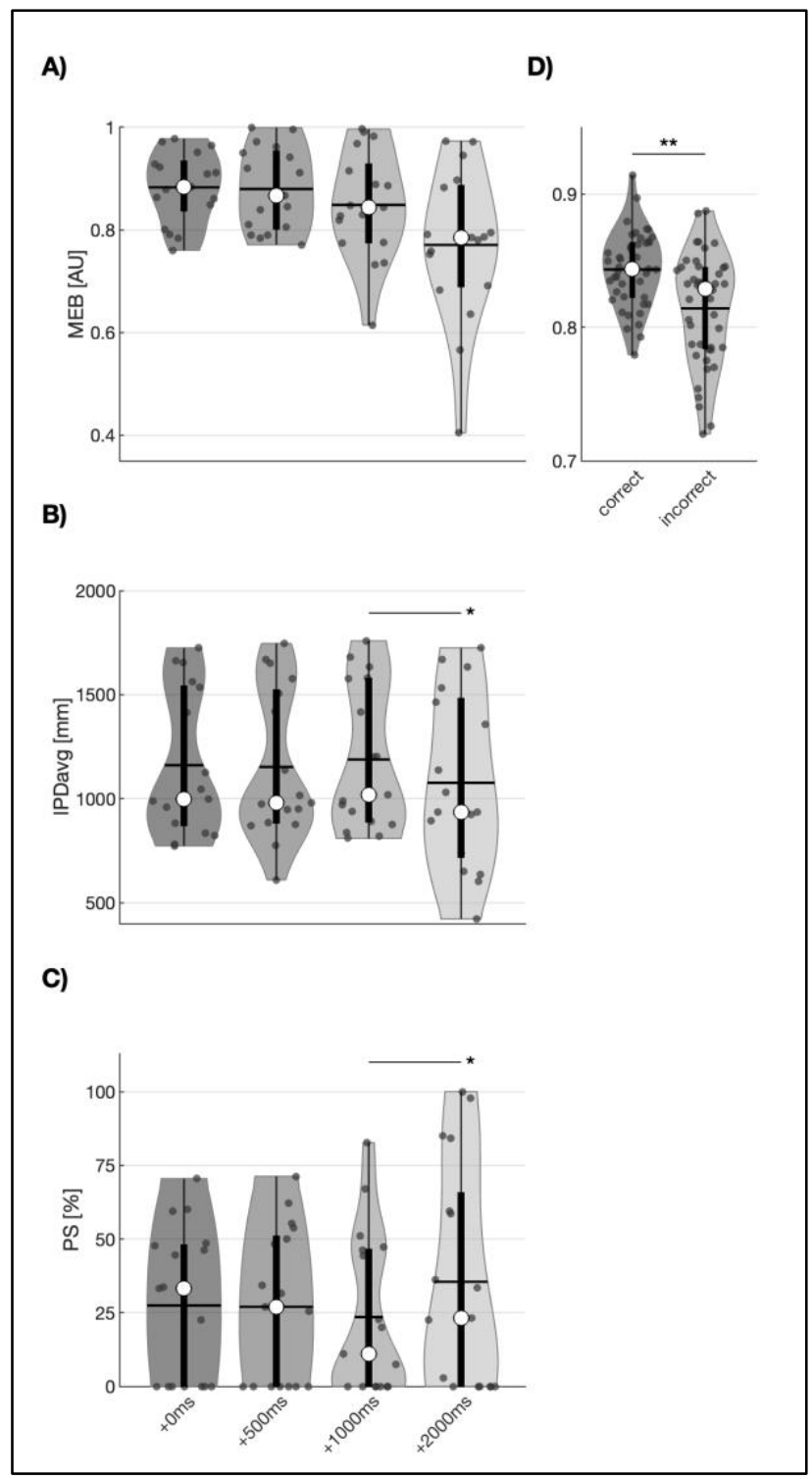

Figure 6: Differences in kinematic parameters. A, B, C: Kinematic differences between temporal offsets ( $+0 \mathrm{~ms},+500 \mathrm{~ms},+1000 \mathrm{~ms},+2000 \mathrm{~ms}$ ) for A) motion energy balance (MEB) in affection sequences, B) average interpersonal distance (IPDavg) in anger sequences, and B) personal space (PS) in anger sequences. Violin plots display the distribution of all sequences (single colored dots represent individual interaction sequences). D: Kinematic differences between correctly and incorrectly identified sequences for motion energy balance in affection sequences. Violin plots display the distribution of mean motion energy balance values (single colored dots represent average values for each subject). Each violin plot contains information about the median (i.e., white dot), the 25th and 75th percentiles (i.e., bold vertical line), and the mean (i.e., horizontal line). Horizontal lines between variables indicate significance of difference between categories. Significance level is indicated by asterisks $(* p<.05, * p<.01)$. 


\section{Discussion}

Human sensitivity to emotional body language has been documented in a broad body of literature (for a review, see de Gelder, 2006) that emphasizes our ability to quickly retrieve the state of a person even if observation is based on highly reduced stimuli. We are able to make a number of inferences that lead to attributions of emotional states and intentions, trigger predictions of others' behaviors, and motivate corresponding adjustments of our own behavior. It is especially physical properties of the observed movements such as spatiotemporal coupling of actions that seem to be a critical source of information for such inferences (see, e.g., Dael et al., 2013).

In the present study, we showed that recognition of emotions from dyadic interactions does, indeed, depend on a fixed spatiotemporal coupling of the actions: Temporal decoupling of one of the agent's actions within an emotional interaction leads to a decrease in recognition performance. However, this is true exclusively for affection and anger sequences. Recognition of happiness and sadness sequences is not impacted significantly by a temporal decoupling. Furthermore, we found that valence perception is also affected in an emotion-specific manner: Affection and anger scenes are perceived as being less intense when the sequences are displayed with a temporal offset. However, there is no change in subjective perception of valence when happiness or sadness sequences are displayed with a temporal offset. These results demonstrate that the temporal decoupling of an agent's actions within an emotional interaction alters the perception of emotional intensity in an observed scene, and - depending on the displayed emotion - this leads to a decrease in emotion recognition performance.

We further explored how the administered temporal offset leads to changes to specific movement features that might explain changes in the subjective impression and recognition of the observed emotional interactions. For affection sequences, we found that motion energy balance between the observed agents decreases linearly with increasing temporal offset. This corresponds directly to the behavioral data. Furthermore, we found that motion energy 
balance is significantly greater for correctly compared to incorrectly identified sequences. With respect to anger sequences, we found changes in the average interpersonal distance and personal space - albeit not corresponding to the behavioral data. In the following, we shall discuss the main findings in detail.

\section{Temporal Coupling and Emotion Recognition}

Within dyadic interactions, it is necessary to be able to adjust one's actions to those of another person. This involves choosing an appropriate complementary action at an appropriate time, and it requires the ability to predict what others will do next - that is, when and how their actions will unfold (Sebanz \& Knoblich, 2009). Even if we are not part of the social interaction ourselves, we seem to possess implicit knowledge about the natural dynamics of the observed human interaction (von der Lühe et al., 2016). Studies on visual discrimination have shown that, with respect to interactive activities, the actions of one agent can be used to predict what the second agent will do (Manera et al., 2011, 2013; Neri et al., 2006; von der Lühe et al., 2016). Manera et al. (2013), for instance, demonstrated that manipulating the timing of the actions of two agents had a detrimental effect on visual discrimination performance only within a communicative condition - that is, a condition in which the gestures performed by one agent were related to those performed by the other agent. In an individual condition in which the actions of the two agents were not related, no effect of timing manipulation was found.

The present findings show that a temporal decoupling of emotional interactions between two agents affects recognition performance for the respective emotion. More specifically, recognition performances decline linearly with increasing temporal offset. Interestingly, this effect is emotion-specific, because the decline of recognition performances is observed only for temporally decoupled affection and anger sequences. Recognition of happiness and sadness is not affected. This suggests that some emotions have a stronger 
interpersonal and communicative character than others and depend more on reciprocal actions between the agents, whereas others may be acted out more on an individual level.

A study by Clarke et al. (2005) underpins this assumption. Within their study, they found that romantic love and joy are recognized more reliably when they are depicted within an interaction (i.e., dyad) instead of individually (i.e., monad). The authors argued that this may be because these emotions are particularly socially expressive and have a stronger interpersonal character. Thus, for certain emotional interactions, the spatiotemporal coupling of the two agents may be of great significance, because we seem to use the relatedness of actions for the prediction, simulation, and inference of the emotional content.

This assumption can be strengthened partly by our exploratory analyses suggesting that the motion energy balance of two agents may play a pivotal role in the recognition of affection scenes. Motion energy balance is a parameter providing information about how much of the total amount of body movement within one sequence can be ascribed to each interactive partner. We found a linear decrease with increasing temporal offset that corresponds directly to the linear decrease in recognition accuracy with increasing temporal offset. Moreover, we found that correctly identified trials are characterized by an increased motion energy balance as compared to incorrectly identified trials. Although these analyses do not allow a causal conclusion, we speculate that recognition accuracy of affection may be facilitated by highly balanced motion energy between the agents. In this vein, Keck et al., (2021) revealed that balance within emotional interactions seems to be an important property for the observer to generate an emotional percept. For example, they found that especially motion energy balance between two agents allows a perceptual distinction between positive and negative valence in observed interactions. Hence, social context information such as motion energy balance is particularly important for recognizing emotional content, especially when the depicted emotions depend on reciprocal actions (Clarke et al., 2005; Kret \& de Gelder, 2010). 
We did not observe a decrease in recognition performance for happiness sequences. This is not in line with the results of Clarke et al. (2005). However, a methodological difference has to be taken into account, because Clarke et al. (2005) presented monads (i.e., single agents) taken from the dyadic interaction. In our case, both agents were depicted, but with different temporal offsets. Thus, in the present study, social context information is continuously available to the observer. This suggests that observers take contextual information into account (i.e., information from the entire scene) when judging the emotional content of a situation. This view is supported by research showing that recognition of emotional expressions is highly dependent on the context of the interaction (Barrett et al., 2019). For instance, by using synthetic dynamic stereotypical expressions, Mumenthaler and Sander (2012) showed that a facial expression was judged as being more fearful when the contextual face, displayed in the periphery of the screen, expressed anger as opposed to fear. Moreover, Abramson et al. (2020) showed that recognition of a fearful body was better when situated with an angry one, but only when the figures were facing each other, thereby creating the impression that they are interacting. Thus, recognition of an emotion seems to operate through functional relations between interactive partners, and it may be specific to the emotion that is displayed. The evidence provided here further suggests that the presence of a second agent is used as an informative contextual cue when attempting to decipher the emotions of others.

Finally, it should be noted that we also found that the recognition of anger deteriorates with increasing temporal offset. Although interpersonal dynamics of angry interactions have not been quantified on an objective level, studies show that anger is usually directed toward someone and requires an immediate action (Grillon \& Charney, 2011). A study by de Valk et al. (2015) asked participants to touch the screen as soon as an image (i.e., emotional or neutral face or body) was displayed. They found that angry body expressions triggered faster actions (as compared to neutral or fearful expressions). The authors interpreted these results in terms 
of an enhanced attention and readiness to act. Although only speculative, introducing a temporal offset delays the reaction of the agent the anger is directed toward, possibly making it more difficult for the perceiver to recognize the respective emotion.

With respect to interpersonal movement parameters, we were unable to identify changes in movement parameters that correspond directly to the behavioral ratings. Although we observed an effect for the average interpersonal distance and the personal space shared by the agents, these effects do not give reason to assume that they drive the behavioral effects caused by the temporal offset.

\section{Temporal Coupling and Subjective Valence Perception}

So far, recognition performance for emotional stimuli has been investigated only with respect to the influence of a temporal decoupling between two interacting agents. However, we were also interested in a more subjective dimension of emotion perception: the perceived emotional valence and intensity. Therefore, we explored how temporal coupling is linked to how negatively or positively a scene is perceived.

We found that valence perception is dependent on a temporal coupling between the actions of two agents. Although there was no overall difference between the original timing and a delay of $+500 \mathrm{~ms}$, the present data reveal that the sequences are perceived as more intense in terms of valence when they are presented in their original form as compared to a delay of +1000 and +2000 ms. This effect, again, is emotion-specific. Whereas valence judgments for sadness and happiness do not differ regardless of the temporal offset, anger and affection trials receive higher valence ratings (i.e., more negative for anger and more positive for affection) and are perceived as more intense in the original timing set.

To the best of our knowledge, the link between temporal coupling and valence perception of emotional body movements has not been investigated so far. Here, we show for the first time that the perception of valence intensity depends on (inter-)actions that occur in a time-locked manner-albeit this is not equally true for all emotion categories. Specifically, 
affection and anger scenes are perceived as more intense when the actions of both agents occur in a temporally and spatially meaningful pattern. In contrast, no effect of timing manipulation is observed when happiness and sadness sequences are displayed. This further supports the notion of the relevance of contextual information.

Taking these results together, time-locked contextual social information seems to enhance the perception of emotional body language and, thereby, might also facilitate emotion recognition.

\section{Temporal Coupling, Movement Contingency, and Action Prediction}

In light of the current results, the question arises why spatiotemporal coupling plays such a fundamental role in the perception of certain emotional interactions. Evidence suggests that the contingency of movement patterns - that is, the temporal and spatial relatedness of movements — facilitates the perception of "meaning" in a visual stimulus (Castelli et al., 2000; Gobbini et al., 2007). Consequently, the term "social contingency" has been used to describe the relatedness of the complex, coordinated actions within dyadic interactions that result in a meaningful movement pattern (Moran et al., 1992). Hence, motion congruency might serve as a fundamental factor in both detecting and understanding complex actions in human interaction. Removing these movement contingencies by delaying the actions of one of the agents, in contrast, results in changes of interaction-specific movement parameters, decreased emotion recognition performance, and also a diminished perception of emotional intensity. This suggests that these contingencies are critical to the perception of emotional dyadic interactions.

What Mechanisms Do Humans Utilize to Understand Affective States When They Are Observing Complex Human Interactions?

Findings regarding action simulation processes suggest that the observation of actions is a predictive activity (Flanagan \& Johansson, 2003; Graf et al., 2007; Verfaillie \& Daems, 2002). When we observe someone's current actions, we automatically anticipate and predict 
their future ones. In a series of behavioral experiments, Graf et al. (2007) demonstrated that we seem to make real-time predictions about how actions will unfold. Besides predicting how an action will unfold, it has been suggested that we also predict when it will unfold. More precisely, we are able to accurately apply temporal predictions generated in our own motor system to observed actions (Sato, 2008; Wilson \& Knoblich, 2005).

For the observation of social interactions, it has been demonstrated that the movements of one agent are used to predict how and when the second agent will respondprovided that the actions of the two agents are related in time (Manera et al., 2013). Thus, it seems plausible that a temporal decoupling will cause a decrease in emotion recognition as well as a diminished perceived intensity of the emotion due to a violation of predicted interaction consequences-however, only under the condition that the interactive movements are functionally related or have a communicative function.

On a neurophysiological level, it is suggested that action prediction within dyadic interactions is rooted in activation of the so-called action observation system (AON; Georgescu et al., 2014). The AON is believed to integrate observed actions of others with an individual's personal motor repertoire, thereby making sense of an action (for a metaanalytical overview, see Caspers et al., 2010). Georgescu et al. (2014) found that the AON is preferentially engaged by contingency within movement patterns when interacting dyads are displayed instead of individually acting monads. The authors argued that the complexity of action representation is determined not merely by the communicative nature of the observed behavior but, indeed, by the relational context in which such behavior is performed. Thus, the AON could be considered to be an early key processing component that supports and contributes to the understanding of nonverbal social interaction; and an automatic movement analysis might be performed to adequately understand an observed agent's social intentions (Gallese, 2006; Gallese \& Goldman, 1998; Jacob \& Jeannerod, 2005). 


\section{Conclusion}

The present findings extend previous evidence on the perception of emotional body language in social interactions by emphasizing the fundamental role of the spatiotemporal coupling of movements within affective dyadic interactions. We show that a valid spatiotemporal coupling between actions is especially necessary for the recognition of anger and affection, but not for happiness or sadness. Moreover, the same effect becomes visible for the perception of the valence intensity of a sequence-however, especially for anger and affection sequences. Hence, our findings suggest that the recognition of emotions seems to operate through functional relations between two agents that are specific to the emotion that is displayed.

\section{Limitations and Future Implications}

With regard to the quantitative movement features, it should be noted that this analysis was conducted post hoc in order to gain a deeper understanding of the observed behavioral effects by describing objective movement properties of our stimuli. Due to its exploratory nature, we are unable to draw causal conclusions from our findings about the effects on behavioral judgments. Thus, future studies should investigate these effects further by systematically varying inter- as well as intrapersonal parameters to see whether and how these affect the recognition of certain emotions.

\section{Data and Code Availability Statement}

The stimulus set, experimental data, and code to reproduce the results presented here are openly available in OSF at https://doi.org/10.17605/OSF.IO/Z3T4J (Bachmann et al., 2021). The SAMI toolbox is archived in Zenodo (Zabicki \& Keck, 2021) as well as openly available on Github (https://github.com/azabicki/SAMI/).

\section{Funding}

This work was supported by the Deutsche Forschungsgemeinschaft (DFG) under a grant (IRTG 1901) for JB and JK. 


\section{Author Contributions}

Bachmann: Conceptualization, Methodology, Validation, Formal analysis, Investigation, Writing - Original Draft, Writing - Review \& Editing, Visualization Krüger: Validation, Writing - Review \& Editing, Supervision Keck: Formal analysis, Writing -

Review \& Editing Munzert: Writing - Review \& Editing, Supervision, Funding acquisition Zabicki: Conceptualization, Methodology, Software, Validation, Formal analysis, Investigation, Data Curation, Writing - Original Draft, Writing - Review \& Editing, Visualization

\section{Decleration of interest}

The authors have no competing interests to declare.

\section{Acknowledgments}

The authors thank Jonathan Harrow for native speaker support. We also thank MarieClaire Englert, Franziska Schulz and Fabian Kowallik for their support during data collection. 


\section{References}

Abramson, L., Petranker, R., Marom, I., \& Aviezer, H. (2020). Social interaction context shapes emotion recognition through body language, not facial expressions. Emotion. https://doi.org/10.1037/emo0000718

Bachmann, J., Krüger, B., Keck, J., Munzert, J., \& Zabicki, A. (2021). Temporal Coupling in Emotional Interactions. https://doi.org/10.17605/OSF.IO/Z3T4J

Bachmann, J., Zabicki, A., Munzert, J., \& Krüger, B. (2020). Emotional expressivity of the observer mediates recognition of affective states from human body movements. Cognition and Emotion, 34(7), 1370-1381. https://doi.org/10.1080/02699931.2020.1747990

Barrett, L. F., Adolphs, R., Marsella, S., Martinez, A. M., \& Pollak, S. D. (2019). Emotional Expressions Reconsidered: Challenges to Inferring Emotion From Human Facial Movements: Psychological Science in the Public Interest. https://doi.org/10.1177/1529100619832930

Becchio, C., Sartori, L., \& Castiello, U. (2010). Toward You: The Social Side of Actions. Current Directions in Psychological Science, 19(3), 183-188. https://doi.org/10.1177/0963721410370131

Beck, A. T., Steer, R. A., \& Brown, G. K. (1996). Beck Depression Inventory-II. Psychological Corporation. https://doi.org/10.1037/t00742-000

Caspers, S., Zilles, K., Laird, A. R., \& Eickhoff, S. B. (2010). ALE meta-analysis of action observation and imitation in the human brain. NeuroImage, 50(3), 1148-1167. https://doi.org/10.1016/j.neuroimage.2009.12.112

Castelli, F., Happé, F., Frith, U., \& Frith, C. (2000). Movement and Mind: A Functional Imaging Study of Perception and Interpretation of Complex Intentional Movement Patterns. NeuroImage, 12(3), 314-325. https://doi.org/10.1006/nimg.2000.0612

Clarke, T. J., Bradshaw, M. F., Field, D. T., Hampson, S. E., \& Rose, D. (2005). The Perception of Emotion from Body Movement in Point-Light Displays of Interpersonal 
Dialogue. Perception, 34(10), 1171-1180. https://doi.org/10.1068/p5203

Dael, N., Goudbeek, M., \& Scherer, K. R. (2013). Perceived Gesture Dynamics in Nonverbal Expression of Emotion. Perception, 42(6), 642-657. https://doi.org/10.1068/p7364 de Gelder, B. (2006). Towards the neurobiology of emotional body language. Nature Reviews Neuroscience, 7(3), 242-249. https://doi.org/10.1038/nrn1872

de Gelder, B., \& Hortensius, R. (2014). The Many Faces of the Emotional Body. In J. Decety \& Y. Christen (Eds.), New Frontiers in Social Neuroscience (Vol. 21, pp. 153-164). Springer. https://doi.org/10.1007/978-3-319-02904-7_9

de Valk, J. M., Wijnen, J. G., \& Kret, M. E. (2015). Anger fosters action. Fast responses in a motor task involving approach movements toward angry faces and bodies. Frontiers in Psychology, 6, 1240. https://doi.org/10.3389/fpsyg.2015.01240

Flanagan, J. R., \& Johansson, R. S. (2003). Action plans used in action observation. Nature, 424(6950), 769-771. https://doi.org/10.1038/nature01861

Gallese, V. (2006). Embodied simulation: From mirror neuron systems to interpersonal relations. In G. Bock \& J. Goode (Eds.), Empathy and Fairness: Novartis Foundation Symposium (Vol. 278). John Wiley.

Gallese, V. (2009). Mirror Neurons, Embodied Simulation, and the Neural Basis of Social Identification. Psychoanalytic Dialogues, 19(5), 519-536. https://doi.org/10.1080/10481880903231910

Gallese, V., \& Goldman, A. (1998). Mirror neurons and the simulation theory of mind-reading. Trends in Cognitive Sciences, 2(12), 493-501. https://doi.org/10.1016/S13646613(98)01262-5

Gallese, V., Keysers, C., \& Rizzolatti, G. (2004). A unifying view of the basis of social cognition. Trends in Cognitive Sciences, 8(9), 396-403. https://doi.org/10.1016/j.tics.2004.07.002

Georgescu, A. L., Kuzmanovic, B., Santos, N. S., Tepest, R., Bente, G., Tittgemeyer, 
M., \& Vogeley, K. (2014). Perceiving nonverbal behavior: Neural correlates of processing movement fluency and contingency in dyadic interactions: Perceiving Nonverbal Interactive Behavior. Human Brain Mapping, 35(4), 1362-1378. https://doi.org/10.1002/hbm.22259

Gobbini, M. I., Koralek, A. C., Bryan, R. E., Montgomery, K. J., \& Haxby, J. V. (2007). Two Takes on the Social Brain: A Comparison of Theory of Mind Tasks. Journal of Cognitive Neuroscience, 19(11), 1803-1814. https://doi.org/10.1162/jocn.2007.19.11.1803

Graf, M., Reitzner, B., Corves, C., Casile, A., Giese, M., \& Prinz, W. (2007). Predicting point-light actions in real-time. NeuroImage, 36, T22-T32. https://doi.org/10.1016/j.neuroimage.2007.03.017

Grillon, C., \& Charney, D. R. (2011). In the face of fear: Anxiety sensitizes defensive responses to fearful faces. Psychophysiology, 48(12), 1745-1752. https://doi.org/10.1111/j.1469-8986.2011.01268.x

Hill, H., \& Pollick, F. E. (2000). Exaggerating Temporal Differences Enhances Recognition of Individuals from Point Light Displays. Psychological Science, 11(3), 223228. https://doi.org/10.1111/1467-9280.00245

Jacob, P., \& Jeannerod, M. (2005). The motor theory of social cognition: A critique. Trends in Cognitive Sciences, 9(1), 21-25. https://doi.org/10.1016/j.tics.2004.11.003

Keck, J., Zabicki, A., Bachmann, J., Munzert, J., \& Krüger, B. (2021). Decoding spatiotemporal features of emotional body language in social interactions. (under review)

Kilner, J. M., Friston, K. J., \& Frith, C. D. (2007). Predictive coding: An account of the mirror neuron system. Cognitive Processing, 8(3), 159-166. https://doi.org/10.1007/s10339-007-0170-2

Kret, M. E., \& de Gelder, B. (2010). Social context influences recognition of bodily expressions. Experimental Brain Research, 203(1), 169-180. https://doi.org/10.1007/s00221$010-2220-8$

Lorey, B., Kaletsch, M., Pilgramm, S., Bischoff, M., Kindermann, S., Sauerbier, I., 
Stark, R., Zentgraf, K., \& Munzert, J. (2012). Confidence in Emotion Perception in PointLight Displays Varies with the Ability to Perceive Own Emotions. PLoS ONE, 7(8), e42169. https://doi.org/10.1371/journal.pone.0042169

Manera, V., Becchio, C., Schouten, B., Bara, B. G., \& Verfaillie, K. (2011).

Communicative Interactions Improve Visual Detection of Biological Motion. PLoS ONE, 6(1), e14594. https://doi.org/10.1371/journal.pone.0014594

Manera, V., Schouten, B., Verfaillie, K., \& Becchio, C. (2013). Time Will Show: Real Time Predictions during Interpersonal Action Perception. PLoS ONE, 8(1), e54949. https://doi.org/10.1371/journal.pone.0054949

Mather, G., \& Murdoch, L. (1994). Gender Discrimination in Biological Motion Displays Based on Dynamic Cues. Proceedings: Biological Sciences, 258(1353), 273-279. https://doi.org/10.1098/rspb.1994.0173

Moran, G., Dumas, J. E., \& Symons, D. K. (1992). Approaches to sequential analysis and the description of contingency in behavioral interaction. Behavioral Assessment, 14(1), $65-92$.

Mumenthaler, C., \& Sander, D. (2012). Social appraisal influences recognition of emotions. Journal of Personality and Social Psychology, 102(6), 1118-1135. https://doi.org/10.1037/a0026885

Neri, P., Luu, J. Y., \& Levi, D. M. (2006). Meaningful interactions can enhance visual discrimination of human agents. Nature Neuroscience, 9(9), 1186-1192. https://doi.org/10.1038/nn1759

Petrini, K., Piwek, L., Crabbe, F., Pollick, F. E., \& Garrod, S. (2014). Look at those two!: The precuneus role in unattended third-person perspective of social interactions: Precuneus and Third-Person Interaction. Human Brain Mapping, 35(10), 5190-5203. https://doi.org/10.1002/hbm.22543

Rindermann, H. (2009). Emotionale-Kompetenz-Fragebogen: EKF; Einschätzung 
emotionaler Kompetenzen und emotionaler Intelligenz aus Selbst-und Fremdsicht. Hogrefe.

Rizzolatti, G., \& Craighero, L. (2004). The mirror-neuron system. Annual Review of Neuroscience, 27, 169-192. https://doi.org/10.1146/annurev.neuro.27.070203.144230

Rosenthal, R. (1994). Parametric Measures of Effect Size. In H. Cooper \& L. V. Hedges (Eds.), The Handbook of Reseach Synthesis (pp. 231-244). Russell Sage Foundation.

Runeson, S., \& Frykholm, G. (1983). Kinematic Specification of Dynamics as an Informational Basis for Person-and-Action Perception: Expectation, Gender Recognition, and Deceptive Intention. 112(4), 585-615. https://doi.org/10.1037/0096-3445.112.4.585

Sato, A. (2008). Action observation modulates auditory perception of the consequence of others' actions. Consciousness and Cognition, 17(4), 1219-1227. https://doi.org/10.1016/j.concog.2008.01.003

Sebanz, N., \& Knoblich, G. (2009). Prediction in Joint Action: What, When, and Where. Topics in Cognitive Science, 1(2), 353-367. https://doi.org/10.1111/j.17568765.2009.01024.x

Spielberger, C. D., Gorsuch, R. L., Lushene, R. E., Vagg, P. R., \& Jacobs, G. A. (1983). Manual for the State-Trait Anxiety Inventory. Consulting Psychologist Press.

Templeton, G. F. (2011). A Two-Step Approach for Transforming Continuous Variables to Normal: Implications and Recommendations for IS Research. Communications of the Association for Information Systems, 28(4), 41-58. https://doi.org/10.17705/1CAIS.02804

Thurman, S. M., \& Lu, H. (2014). Perception of Social Interactions for Spatially Scrambled Biological Motion. PLoS ONE, 9(11), e112539. https://doi.org/10.1371/journal.pone.0112539

Troje, N. F. (2002). Decomposing biological motion: A framework for analysis and synthesis of human gait patterns. Journal of Vision, 2(5), 2. https://doi.org/10.1167/2.5.2 Verfaillie, K., \& Daems, A. (2002). Representing and anticipating human actions in 
vision. Visual Cognition, 9(1-2), 217-232. https://doi.org/10.1080/13506280143000403

von der Lühe, T., Manera, V., Barisic, I., Becchio, C., Vogeley, K., \& Schilbach, L. (2016). Interpersonal predictive coding, not action perception, is impaired in autism.

Philosophical Transactions of the Royal Society B: Biological Sciences, 371(1693), 20150373. https://doi.org/10.1098/rstb.2015.0373

Wicker, B., Keysers, C., Plailly, J., Royet, J.-P., Gallese, V., \& Rizzolatti, G. (2003). Both of Us Disgusted in My Insula: The Common Neural Basis of Seeing and Feeling Disgust. Neuron, 40(3), 655-664. https://doi.org/10.1016/S0896-6273(03)00679-2

Wilson, M., \& Knoblich, G. (2005). The Case for Motor Involvement in Perceiving Conspecifics. 131(3), 460-473. https://doi.org/10.1037/0033-2909.131.3.460

Zabicki, A., \& Keck, J. (2021). SAMI: Similarity Analysis of Human Movements and Interactions. Zenodo. https://doi.org/10.5281/zenodo.4764552 


\section{Supplementary Material}

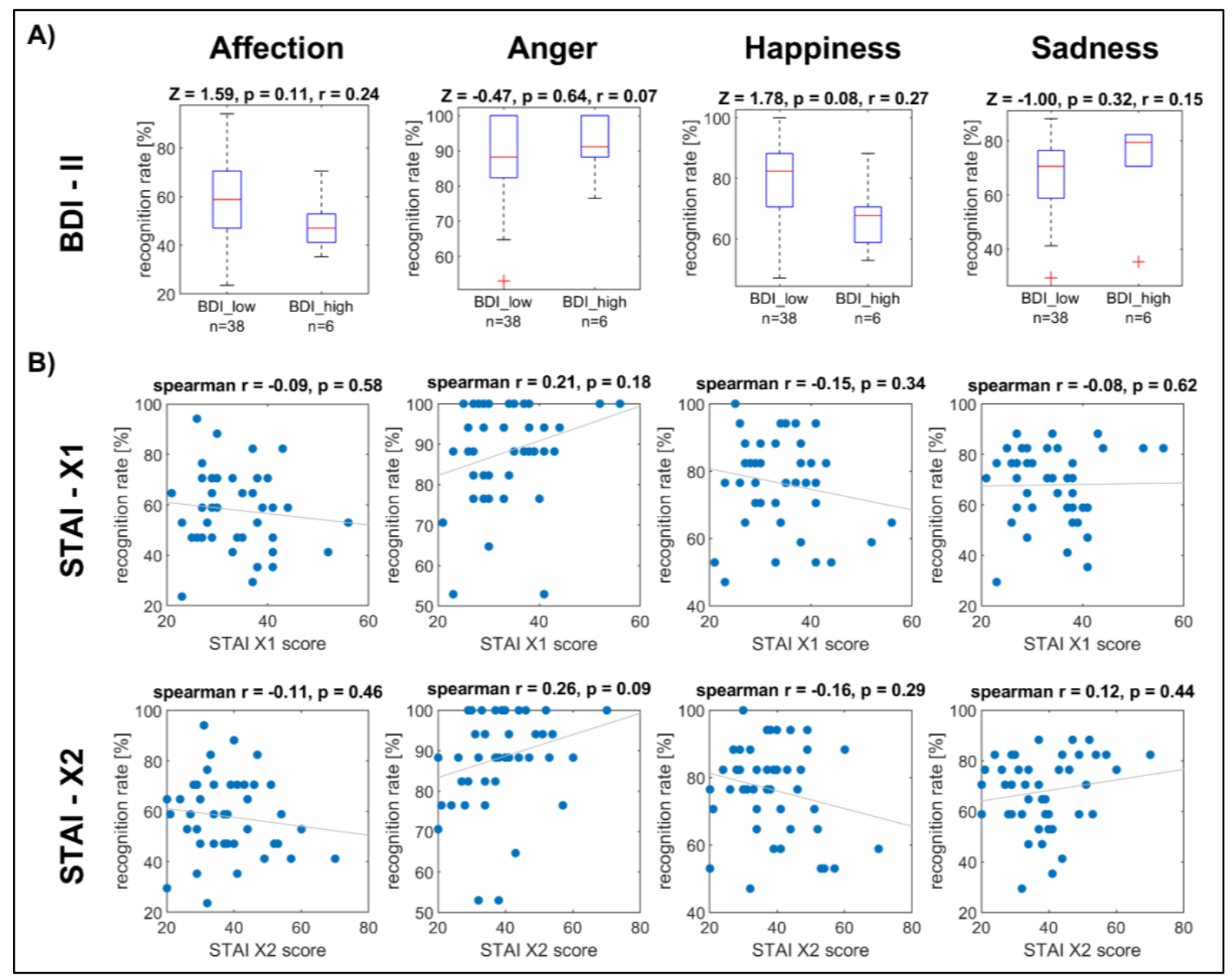

Figure S1. Controlling for the influence of BDI-II and STAI scores. A) Nonparametric MannWhitney $U$ tests indicated no significant differences in recognition rates (see Manuscript Figure 2B) between participants with a total BDI-II score of a value below or equal to 13 ( $n=$ $38)$ and those with a value above $13(n=6)$. B) Spearman correlation coefficients indicating no significant relationships between participants' STAI X1 and X2 scores and their corresponding emotion recognition rates. 


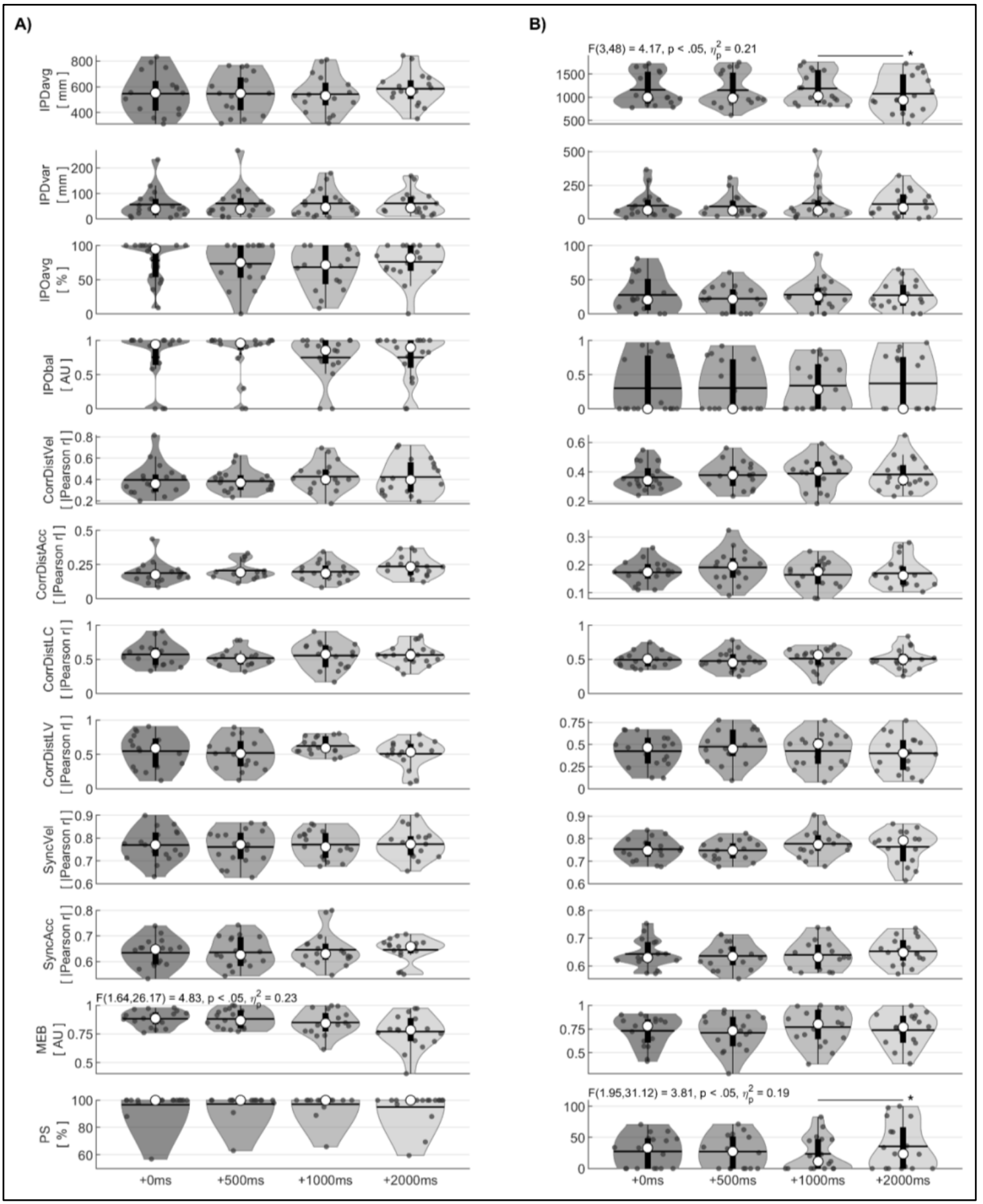

Figure S2. Influence of temporal offset on kinematic features. Differences between temporal offsets (+0 ms, $+500 \mathrm{~ms},+1000 \mathrm{~ms},+2000 \mathrm{~ms}$ ) displayed for each kinematic feature separated by A) affection sequences and B) anger sequences. Violin plots display the distribution of all sequences (single colored dots represent individual interaction sequences). Each violin plot contains information about the median (i.e., white dot), the 25th and 75 th percentiles (i.e., bold vertical line), and the mean (i.e., horizontal line). ANOVA statistics are displayed for significant main effects. Horizontal lines between variables indicate significance of difference between categories. Significance level is indicated by asterisks $\left({ }^{*} p<.05\right)$. 
Table S1.

Summary of Interaction-Specific Kinematic Features Calculated by the SAMI Toolbox*

\begin{tabular}{lll}
\hline Kinematic interaction feature & Abbr. & Short description \\
\hline Average of Interpersonal Distance & IPDavg & $\begin{array}{l}\text { Spatial distance between both agents. Calculated as } \\
\text { the mean over time, respectively the standard } \\
\text { deviation. }\end{array}$ \\
\hline Variance in Interpersonal Distance & IPDvar & \\
\hline
\end{tabular}

Average of Interpersonal

Orientation

IPOavg

Balance of Interpersonal

Orientation

IPObal
Time spent facing each other + time spent by one agent facing the other and vice versa.

\begin{tabular}{lll}
\hline $\begin{array}{l}\text { Correlation between Spatial } \\
\text { Distance and Velocity }\end{array}$ & CorrDistVel & $\begin{array}{l}\text { Absolute correlation between spatial distance and } \\
\text { mean velocity of whole-body movements }\end{array}$ \\
\hline $\begin{array}{l}\text { Correlation between Spatial } \\
\text { Distance and Acceleration }\end{array}$ & CorrDistAcc & $\begin{array}{l}\text { Absolute correlation between spatial distance and } \\
\text { mean acceleration of whole-body movements }\end{array}$ \\
$\begin{array}{l}\text { Correlation between Spatial } \\
\text { Distance and Limb Contraction }\end{array}$ & CorrDistLC & $\begin{array}{l}\text { Absolute correlation between spatial distance and } \\
\text { limb posture/movement }\end{array}$ \\
\hline $\begin{array}{l}\text { Correlation between Spatial } \\
\text { Distance and Limb Volume }\end{array}$ & CorrDistLV & $\begin{array}{l}\text { Absolute correlation between spatial distance and } \\
\text { the space taken up by the three-dimensional total } \\
\text { body extension }\end{array}$ \\
\hline
\end{tabular}

\begin{tabular}{lll} 
Synchronization of Velocity & SyncVel & $\begin{array}{l}\text { Absolute correlation between the agent's velocity } \\
\text { profiles }\end{array}$ \\
\hline $\begin{array}{l}\text { Synchronization of Acceleration } \\
\text { Motion Energy Balance }\end{array}$ & SyncAcc & $\begin{array}{l}\text { Absolute correlation between the agent's } \\
\text { acceleration profiles }\end{array}$ \\
\hline Personal Space & PS & $\begin{array}{l}\text { Total amount of body movement over time that can } \\
\text { be ascribed to each interactive partner }\end{array}$ \\
\hline
\end{tabular}

* For a detailed description of each kinematic feature and how they are calculated, see Zabicki \& Keck (2021). 
Table S2.

Results of Repeated Measures ANOVAs Comparing Differences in Kinematic Features With Respect to Temporal Offsets

\begin{tabular}{|c|c|c|c|c|c|c|c|c|c|c|c|}
\hline \multirow{2}{*}{$\begin{array}{l}\text { Kinematic } \\
\text { Feature }\end{array}$} & \multirow{2}{*}{ Predictor } & \multicolumn{5}{|c|}{ Affection } & \multicolumn{5}{|c|}{ Anger } \\
\hline & & Epsilon & $d f$ & $\boldsymbol{F}$ & $p$ & $\eta_{p}^{2}$ & Epsilon & $d f$ & $\boldsymbol{F}$ & $p$ & $\eta_{p}^{2}$ \\
\hline \multirow[t]{2}{*}{ IPDavg } & Temporal offset & .60 & 1.8 & 2.646 & .09 & .142 & & 3 & 4.168 & $<.05$ & .207 \\
\hline & error & & 28.79 & & & & & 48 & & & \\
\hline \multirow[t]{2}{*}{ IPDvar } & Temporal offset & .55 & 1.65 & .049 & .93 & .003 & .69 & 2.08 & 1.233 & .31 & .072 \\
\hline & error & & 26.45 & & & & & 33.27 & & & \\
\hline \multirow[t]{2}{*}{ IPOavg } & Temporal offset & & 3 & 1.525 & .22 & .087 & & 3 & 1.295 & .29 & .075 \\
\hline & error & & 48 & & & & & 48 & & & \\
\hline \multirow[t]{2}{*}{ IPObal } & Temporal offset & & 3 & .367 & .78 & .022 & .71 & 2.14 & .698 & .51 & .042 \\
\hline & error & & 48 & & & & & 34.21 & & & \\
\hline \multirow[t]{2}{*}{ CorrDistVel } & Temporal offset & & 3 & .968 & .42 & .057 & .54 & 1.62 & .261 & .73 & .016 \\
\hline & error & & 48 & & & & & 25.95 & & & \\
\hline \multirow[t]{2}{*}{ CorrDistAcc } & Temporal offset & & 3 & 2.012 & .12 & .112 & & 3 & 1.322 & .28 & .076 \\
\hline & error & & 48 & & & & & 48 & & & \\
\hline \multirow[t]{2}{*}{ CorrDistLC } & Temporal offset & & 3 & 1.184 & .33 & .069 & & 3 & .335 & .80 & .020 \\
\hline & error & & 48 & & & & & 48 & & & \\
\hline \multirow[t]{2}{*}{ CorrDistLV } & Temporal offset & & 3 & 1.148 & .34 & .067 & & 3 & .508 & .68 & .031 \\
\hline & error & & 48 & & & & & 48 & & & \\
\hline \multirow[t]{2}{*}{ SyncVel } & Temporal offset & & 3 & .198 & .90 & .012 & & 3 & 1.545 & .21 & .088 \\
\hline & error & & 48 & & & & & 48 & & & \\
\hline \multirow[t]{2}{*}{ SyncAcc } & Temporal offset & .61 & 1.82 & .597 & .54 & .036 & & 3 & .540 & .66 & .033 \\
\hline & error & & 29.04 & & & & & 48 & & & \\
\hline \multirow[t]{2}{*}{ MEB } & Temporal offset & .55 & 1.64 & 4.833 & $<.05$ & .232 & & 3 & .698 & .56 & .042 \\
\hline & error & & 26.17 & & & & & 48 & & & \\
\hline \multirow[t]{2}{*}{ PS } & Temporal offset & .55 & 1.66 & .925 & .39 & .055 & .65 & 1.95 & 3.808 & $<.05$ & .192 \\
\hline & error & & 26.62 & & & & & 31.12 & & & \\
\hline
\end{tabular}

Note. A reported Epsilon indicates a Greenhouse-Geisser correction due to lack of sphericity indicated by a significant Mauchly's sphericity test. 
Table S3.

Results of Wilcoxon Signed-Ranks Tests Comparing Kinematic Features Between Correctly and Incorrectly Identified Emotional Sequences

\begin{tabular}{lccc}
\hline \multirow{2}{*}{ Kinematic feature } & \multicolumn{3}{c}{ Affection } \\
\cline { 2 - 4 } & $\boldsymbol{Z}$ & $\boldsymbol{p}$ & $\boldsymbol{r}$ \\
\hline IPDavg & -5.532 & $<.001$ & .958 \\
IPDvar & -2.999 & $<.01$ & .519 \\
IPOavg & 3.839 & $<.001$ & .665 \\
IPObal & 3.641 & $<.001$ & .63 \\
CorrDistVel & -.035 & .97 & .006 \\
CorrDistAcc & 3.396 & $<.001$ & .588 \\
CorrDistLC & -1.155 & .25 & .200 \\
CorrDistLV & 4.330 & $<.001$ & .749 \\
SyncVel & 5.403 & $<.001$ & .935 \\
SyncAcc & 4.166 & $<.001$ & .721 \\
MEB & 3.256 & $<.01$ & .564 \\
PS & 4.995 & $<.001$ & .865 \\
\hline
\end{tabular}

Note. Effect size $r$ is calculated by means of a matched-pairs rank-biserial correlation. 